\title{
Facilitadores y barreras que enfrentan las personas al tomarse el test de ELISA para el diagnóstico del VIH: revisión de la literatura
}

\author{
Alejandra X. Araya, Paulina Bravo, Paola Carrasco, M. Teresa Urrutia, \\ Paula Vega, Miriam Rubio y M. Jesús Lira
}

\begin{abstract}
Pontificia Universidad Católica de Chile, Santiago. Académicos Escuela de Enfermería (AXA, PB, PC, MTU, PV, MR) Estudiante de Enfermería, (MJL).
\end{abstract}

Recibido: 3 de mayo de 2013 Aceptado: 7 de septiembre de 2013

Proyecto financiado por el Fondo Nacional de Investigación en Salud CONICYT-MINSAL, CHILE. Proyecto Fonis SA1112137. La organización que proporcionó el financiamiento no tuvo influencia en el diseño del estudio; en la recolección, análisis o interpretación de los datos; en la preparación, revisión o aprobación del manuscrito

Correspondencia a: Alejandra Ximena Araya aarayagu@puc.cl

\section{Introducción}

$\mathrm{E}$ 1 diagnóstico tardío de la infección por virus de la inmunodeficiencia humana (VIH) es un problema universal ${ }^{1}$. Estadísticas señalan que entre 22 y $83 \%$ de las personas infectadas por VIH fueron diagnosticadas en etapa de SIDA en Europa y Estados Unidos de Améri$\mathrm{ca}^{2-6}$. Esta tendencia se mantiene en Chile, en el año 2008, 9.193 personas fueron diagnosticadas en etapa de $\mathrm{SIDA}^{7}$, representando $46 \%$ de las infectadas por $\mathrm{VIH}^{8}$.

El diagnóstico precoz de la infección por VIH tiene beneficios tanto para las personas como para la salud pública $^{9}$. Para las personas infectadas, existe un aumento en la tasa de sobrevida, disminución de la tasa de morbilidad asociada al $\mathrm{VIH}^{10-12} \mathrm{y}$, por ende, un aumento en su calidad de vida. Para la salud pública, el diagnóstico oportuno es crítico para disminuir el costo económico derivado de las co-morbilidades de la enfermedad ${ }^{10,11,13-15}$ y para prevenir la transmisión del VIH a personas seronegativas ${ }^{16,17}$. La tasa de transmisión es 3,5 veces mayor en el grupo de personas que no conocen su diagnóstico de $\mathrm{VIH}^{18}$.

En Chile, a pesar de la disponibilidad del test de ELISA para el diagnóstico de infección por VIH en la atención primaria de salud, los usuarios continúan tomándoselo tardíamente ${ }^{7}$. El objetivo de esta revisión es conocer cuáles son los factores que facilitan o dificultan que las personas accedan oportunamente a realizarse el test de ELISA para el diagnóstico de infección por VIH en la atención primaria de salud.

\section{Metodología}

Para la búsqueda de los artículos de investigación fueron seleccionadas las siguientes bases de datos: The Cumulative Index to Nursing and Allied Health Literature (CINAHL), The American Psychological Association's PsycINFO ${ }^{\circledR}$, Pub Med and Scientific Electronic Library Online SciELO databases. Estas bases de datos fueron escogidas porque cuentan con los recursos más completos en salud, psicológica y la literatura sudamericana.

Los criterios de inclusión para la selección de los artículos fueron:

- Estudios observacionales, de intervención y cualitativos que describan los elementos facilitadores y/o barreras para la toma oportuna del test de ELISA para el diagnóstico de la infección por VIH, en la atención primaria de salud.

- Artículos publicados en idioma inglés, español o portugués. 
- Publicados en los últimos 10 años (enero de 2001 hasta octubre de 2012), y

- que consideren en la muestra sólo participantes adultos.

Los criterios de exclusión de artículos de investigación de este protocolo de selección fueron:

- La atención pediátrica o maternal.

- Investigaciones realizadas en el servicio de hospitalizados o urgencia.

- El acceso sólo a los resúmenes, y

- revisiones bibliográficas.

La búsqueda en las bases de datos señaladas anteriormente utilizó los siguientes descriptores (HIV OR HUMAN IMMUNODEFICIENCY VIRUS) AND (ELISA OR test*) AND decision* AND (barrier* OR facilitator*). Se ocuparon los recursos de información bibliográfica de la Pontificia Universidad Católica de Chile para la extracción de los artículos de investigación según protocolo de selección.

Las secciones de los artículos de investigación utilizada para el análisis fueron: Métodos, Resultados y Conclusiones. Siguiendo los principios de análisis de contenido, se incluyó la identificación de los significados que se mencionaban de forma más repetitiva, la consistencia de éstos, las explicaciones y relaciones de éstos a través de todos los estudios incluidos en esta revisión.

\section{Resultados}

La búsqueda bibliográfica inicial arrojó 195 títulos. Dos investigadores analizaron independientemente los títulos y resúmenes obtenidos, excluyendo 168 artículos por no cumplir con los criterios de inclusión. Veintisiete artículos a texto completo fueron recuperados, de los cuales 12 de ellos ( 2 revisiones de literatura, 4 tesis y 6 artículos de investigación) fueron excluidos debido a que coincidían con los criterios de exclusión de esta revisión. Por consiguiente, un total de 15 artículos de investigación fueron incluidos en el análisis de esta revisión (Figura 1). La mayoría de los artículos incluidos en esta revisión fue mayoritariamente estudios cualitativos $(n=10)$. Tres estudios eran cuantitativos y dos reportaron método mixto. La mayoría de los estudios fue realizada en África (n: 10) y el resto en América del Norte.

Después de analizar los artículos seleccionados, los resultados se agruparon en dos grandes temas: elementos facilitadores y elementos obstaculizadores para la toma del test de ELISA para el diagnóstico del VIH en la atención primaria de salud. Cada tema hace referencia a factores personales, de los profesionales y de los centros de salud (Figura 2).

\section{Elementos facilitadores para la toma del examen del VIH}

\section{Factores personales}

Conductas de riesgo. El hecho que las personas reconozcan haber realizado una acción riesgosa para adquirir el VIH es un factor que les motiva a tomarse

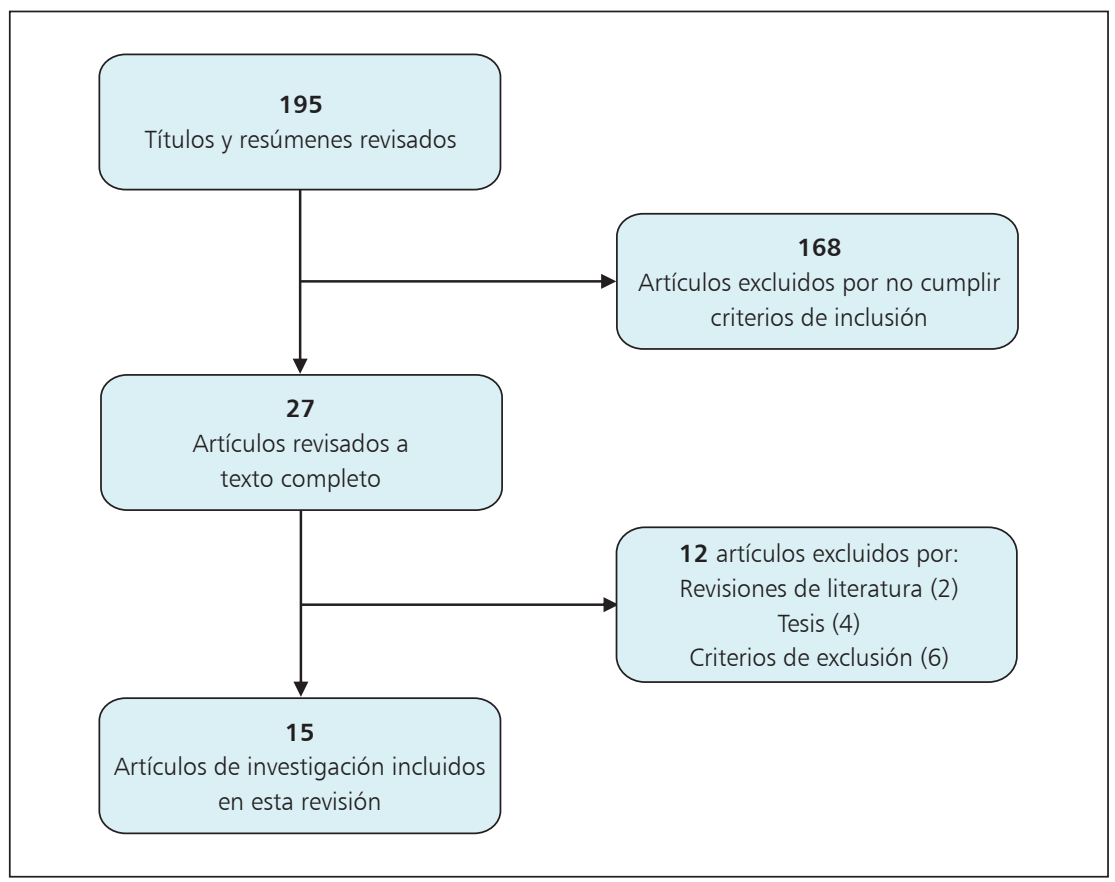

Figura 1. Flujograma de inclusión y exclusión de artículos.

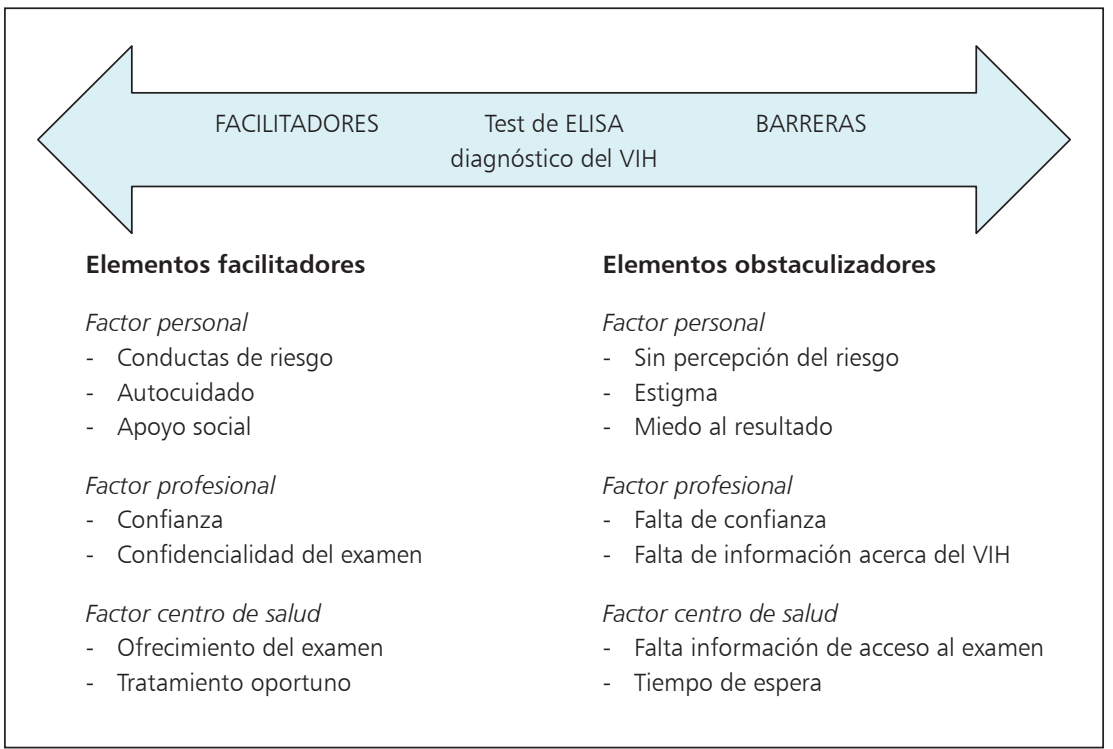

Figura 2. Resumen de elementos facilitadores y obstaculizadores para la toma del test de ELISA para el diagnóstico de infección por VIH. 
el examen. Por ejemplo, un estudio canadiense que involucró a jóvenes aborígenes, señaló que el mantener relaciones sexuales sin preservativo es una de las razones por las que las personas deciden realizarse el examen ${ }^{19}$. En la misma línea, un estudio con estudiantes de origen chino, concluyó que una de las razones más comunes para la realización del examen fue el haber mantenido una relación sexual desprotegida ${ }^{20}$. Estos hechos han sido descritos como eventos precipitantes para la decisión de tomarse el examen del $\mathrm{VIH}^{21,22}$.

Autocuidado. El auto-cuidado es una forma de cuidarse a sí mismo y es otro elemento que favorece que las personas accedan a tomarse el test del VIH. Un estudio realizado en Camerún a 21 mujeres con tuberculosis concluye que las personas que tienen una posición favorable hacia el examen para el VIH son aquellas que tienen deseo de ser saludables y vivir por un mayor tiempo ${ }^{23}$. A sí mismo, en Tanzania, la enfermedad de un hijo o de la pareja hacen a las mujeres tomar la decisión de realizarse el examen ${ }^{24}$.

Apoyo social. El apoyo familiar y/o redes de apoyo aparece como un elemento motivacional a la hora de decidir si realizarse o no el examen. La sugerencia de hacerse el examen de alguna persona cercana a su núcleo familiar o de amigos cercanos es un factor precipitante para tomar la decisión de realizarse el examen. Otro factor encontrado en la literatura médica es la percepción de contar con redes de apoyo una vez conocido el resultado del mismo. Ambos factores se trasforman en elementos fundamentales para tomar la decisión de realizarse el test de ELISA para el diagnóstico de la infección por $\mathrm{VIH}^{22-24}$.

\section{Factores de los profesionales}

Confianza. Estudios señalan que es fundamental que se genere una relación de confianza entre los profesionales y los usuarios, en especial durante las consejerías, a través de un trato amistoso o familiar hacia el usuario, la des-estigmatización del VIH y la entrega de información oportuna y adecuada ${ }^{25,26}$.

Confidencialidad del examen. Que los profesionales de la salud refuercen el concepto de que el examen se realiza de manera reservada, manteniendo durante todo el proceso la privacidad y confidencialidad de éste, es crítico para facilitar la accesibilidad del examen. En los centros de salud, el explicitar que el proceso de toma del test de ELISA para el diagnóstico del VIH es privado y confidencial, es clave para que las personas decidan acceder al examen del $\mathrm{VIH}^{21}$.

\section{Factores organizacionales}

Ofrecimiento del examen. Para que los usuarios dispongan del test de ELISA para el diagnóstico de infección por VIH, es necesario visibilizar y dar a conocer la toma del test al interior del centro de salud. Un estudio cualitativo realizado con parejas a quienes se les invitó a participar de una consejería y posteriormente tomarse el examen en sus casas, demostró que existe una mejor aceptación a acceder la toma del examen a quienes se les ofreció directamente el test ${ }^{25}$.

Tratamiento oportuno. La posibilidad de acceso a un tratamiento oportuno se transforma en un elemento facilitador para acceder al test del VIH. Un estudio mixto realizado con 421 trabajadores sexuales en Guinea, señala que la posibilidad de acceder a tratamiento oportuno aumenta la aceptabilidad del examen entre las personas ${ }^{22}$. Similares resultados se describen en una serie de 12 grupos focales con mujeres y hombres en Uganda, donde el examen era considerado como la oportunidad de acceder a tratamiento y apoyo gratuito de organizaciones para el $\mathrm{VIH}^{27}$.

\section{Elementos que dificultan la toma del examen del VIH}

\section{Factores personales}

Falta de percepción de riesgo. Contrariamente a aquellos que se perciben como en riesgo de adquirir el VIH, las personas que consideran tener un bajo riesgo de ser infectados por el virus, manifiestan no tener la necesidad de realizarse el examen ${ }^{20}$. Un estudio mixto con jóvenes canadienses mostró que $45 \%$ de los participantes reconoció este bajo riesgo como una de las razones principales para no realizarse el examen, mientras que 35\% se negó al examen porque no habían mantenido relaciones sexuales con una persona con serología positiva ${ }^{28,29}$. Los investigadores reportan que esta percepción de invulnerabilidad fue una de las razones más importantes para decidir no realizarse el examen ${ }^{19}$.

Estigma. El miedo a la estigmatización y consecuente discriminación, aparece como uno de los elementos que más dificultan el acceso al examen del test de ELISA para el diagnóstico del VIH. Por ejemplo, las personas reconocen que el estigma que significa el estar infectado por VIH es una limitante para solicitar el examen, dado que esto implica revelar que son personas que están en riesgo de tener serología positiva para $\mathrm{VIH}^{21,30,31}$. A sí mismo, un estudio cualitativo realizado en Zambia con 34 usuarios y 10 consejeros para el examen, mostró que las personas prefieren evitar la "etiqueta" de infectado por VIH y no realizarse el examen ${ }^{30}$. Otro estudio realizado en mujeres canadienses, señala que el miedo a ser estigmatizado no sólo es hacia su propia persona, sino también hacia a sus familias ${ }^{26}$.

Adicionalmente, un estudio realizado con más de 9.000 zimbabuenses señaló que hombres y mujeres que reportaron niveles de estigma hacia personas con infección por VIH, tenían significantemente un número menor de serologías para VIH realizadas ${ }^{32}$. 
Miedo al resultado del examen. Otra de las barreras identificadas por un estudio cualitativo en E.U.A., que involucró a 100 participantes, develó que el miedo al resultado del examen es un motivo por el cual las personas no acceden a éste, lo que se relaciona además con el miedo a transmitir el virus a otros y el inminente pensamiento de muerte por la adquisición del $\mathrm{VIH}^{29}$.

\section{Factores profesionales}

Falta de confianza. La confianza es la seguridad o esperanza de que los profesionales que trabajan en el centro manejarán en reserva los nombres de los usuarios que se han tomado el test de ELISA para el diagnóstico del VIH. Cuando los usuarios reconocen a las organizaciones y los profesionales de la salud como poco confiables, porque pueden poner en riesgo la privacidad y confidencialidad del proceso de toma del test al resto de la comunidad, tienden a rechazar la realización del mismo, especialmente si esta información puede ser conocida por la comunidad ${ }^{23,33}$.

Falta información sobre el VIH. La carencia de entrega de contenidos educativos sobre los factores de riesgo para adquirir el VIH o sobre los servicios existentes en caso de que el resultado del examen sea positivo ha sido considerada como una barrera para acceder oportunamente al test de ELISA para el diagnóstico de infección por VIH. Es así como, un estudio cualitativo realizado en Sudáfrica, con 21 usuarios del sistema de salud, señala que la falta de información sobre el VIH durante la toma del examen puede afectar negativamente el acceso futuro al mismo ${ }^{31}$.

\section{Factores del centro de salud}

Falta de información del acceso al examen. La falta de información o desconocimiento de los usuarios sobre la disponibilidad del test en los centros de salud fue descrita como una de las principales barreras que dificultan el acceso al examen por más de un tercio de los participantes en un estudio en E.U.A. ${ }^{20}$. Además, concluye que servicios de salud no acogedores y profesionales poco empáticos y que presionan a las personas, son aspectos que pueden influir negativamente en la voluntariedad de la toma del examen $^{31}$. Estos resultados concuerdan con los hallazgos reportados por un estudio cualitativo realizado con mujeres y trabajadores de la salud en Canadá ${ }^{26}$.

Tiempo de espera. Un estudio reporta que el tiempo de espera para la toma del examen y la recolección de los resultados es un aspecto que obstaculiza la decisión de realizarse el examen. Este período de espera aumenta la tensión y ansiedad que las personas experimentan en este proceso lo que dificulta la decisión ${ }^{29}$.

\section{Conclusiones}

Esta revisión de la literatura permite describir elementos individuales de los profesionales y de los centros de salud que facilitan o dificultan la decisión de realizarse el test de ELISA para el diagnóstico de infección por VIH. En relación a las personas, el estigma y el miedo a la discriminación aparecen como una de las razones fundamentales para que las personas posterguen esta decisión. El estigma ha sido identificado como un elemento central en la toma de decisiones de personas infectadas por VIH, en relación a la revelación de un diagnóstico seropositivo, el acceso a tratamiento y la mantención de conductas sexuales saludables ${ }^{34}$.

La percepción de riesgo es reconocida como uno de los elementos facilitadores para la toma del examen, pero el riesgo de ser estigmatizado limita esta decisión ${ }^{35,36}$. Esta revisión actualizada de la evidencia permite identificar aquellos elementos de los centros de salud que facilitan o dificultan la decisión de realizarse el examen, lo que resulta una contribución para el desarrollo de intervenciones que apoyen a las personas cuando se enfrentan a esta decisión. Estos elementos coinciden con lo expuesto en un estudio mexicano de población seropositiva para VIH, donde el miedo al diagnóstico y estigma y problemas en el acceso al examen fueron las mayores dificultades para acceder a un diagnóstico oportuno ${ }^{37}$. Recursos y esfuerzos son necesarios para hacer el test del VIH más accesible a la comunidad, reduciendo las barreras para acceder oportunamente al examen, aumentando las motivaciones y razones de las personas para tomarse el test $\mathrm{y}$, consecuentemente, para que más personas pueden conocer su serología oportunamente ${ }^{38}$.

Una de las limitaciones de esta revisión radica en que los artículos incluidos provienen mayormente de África. Las diferencias en relación al acceso al tratamiento dificultan la extrapolación de los resultados de esta revisión. Por ejemplo, aquellos lugares que no garantizan el tratamiento de personas portadoras del VIH podrían presentar una barrera institucional más acentuada para la toma del examen del VIH que otros países que pueden acceder gratuitamente al tratamiento anti-retroviral. Sin embargo, a pesar que Chile cuenta con acceso gratuito a la terapia anti-retroviral, se mantiene una tendencia al diagnóstico tardío del VIH, evidenciando que no sólo la garantía de gratuidad a la terapia es un factor que influye en el acceso al test de ELISA para el diagnóstico del VIH. En este sentido aspectos psicológicos de la enfermedad como miedo y temor al diagnóstico ${ }^{39}$, emergen como importantes barreras a considerar. Además, considerando las particularidades que la pandemia de la infección por VIH tiene en regiones como América Latina, parece importante enfocar los esfuerzos en entender cómo los hallazgos de esta revisión explican los elementos que facilitan o dificultan el acceso al examen del VIH en la región y así intervenir oportunamente en ellos.

Es importante destacar que aquellas personas que presentan un diagnóstico en etapas avanzadas de la en- 
fermedad y, por ende, acceden tardíamente a tratamiento, tendrán que enfrentar un costo de cuidado significativamente mayor que aquellos que fueron diagnosticados oportunamente ${ }^{15}$. Es necesario desarrollar investigaciones orientadas a explorar los aspectos facilitadores y obstaculizadores para el acceso oportuno al test de ELISA para el diagnóstico de infección por VIH en la atención primaria de salud en nuestro país. Esta información es una piedra fundamental para la creación de políticas públicas, que permitan diseñar e implementar estrategias destinadas a aumentar el número de personas que accedan a solicitar el test de ELISA para detectar la infección por VIH en la atención primaria de salud.

\section{Resumen}

Introducción: El diagnóstico tardío de la infección por VIH es un problema universal. A pesar de la disponibilidad del test de ELISA para el diagnóstico de esta infección en la atención primaria de salud, las personas continúan tomándoselo tardíamente. Objetivo: Conocer los factores que facilitan o dificultan que las personas accedan oportunamente al examen en la atención primaria de salud. Métodos: Cuatro bases de datos del área de la enfermería, psicológica, salud biomédica y profesiones afines (años 2001-2012) fueron examinadas con un protocolo de revisión. Resultados: De 195 artículos detectados, 15 cumplieron con los criterios de inclusión y fueron agrupados en dos grandes temas: elementos facilitadores y elementos obstaculizadores de la persona, de los profesionales y de los centros de atención primaria de salud. Percepción de conductas de riesgo, autocuidado, apoyo social, la confianza, confidencialidad del examen, el ofrecimiento del examen y el conocimiento de un tratamiento oportuno han sido reconocidos como uno de los elementos facilitadores para la toma del examen. La falta de información sobre el test y la enfermedad son reconocidas como los principales obstaculizadores para acceder al test. Discusión: La información obtenida es un pilar fundamental para diseñar e implementar estrategias destinadas a aumentar el número de personas que solicitan voluntariamente al examen.

\section{Referencias bibliográfícas}

1.- Valdiserri R O. Late HIV diagnosis: bad medicine and worse public health. PLoS Med 2007; 4 (6): e200. Epub 2007/06/15.

2.- Ndiaye B, Salleron J, Vincent A, Bataille P, Bonnevie F, Choisy P, et al. Factors associated with presentation to care with advanced HIV disease in Brussels and Northern France: 1997-2007. BMC Infect Dis 2011;11:11. Epub 2011/01/14

3.- Yazdanpanah Y, Lange J, Gerstoft J, Cairns G. Earlier testing for HIV-how do we prevent late presentation? Antivir Ther 2010; 15 (Suppl 1): 17-24. Epub 2010/05/14.

4.- Mayben J K, Kramer J R, Kallen M A, Franzini L, Lairson D R, Giordano T P. Predictors of delayed HIV diagnosis in a recently diagnosed cohort. AIDS Patient Care STDS 2007; 21 (3): 195-204. Epub 2007/04/13.

5.- Rotily M, Bentz L, Pradier C, Obadia Y, Cavailler P. Factors related to delayed diagnosis of HIV infection in southeastern France. EVALVIH group. Int J STD AIDS 2000; 11 (8): 531-5. Epub 2000/09/16.

6.- Couturier E, Schwoebel V, Michon C, Hubert J B, Delmas M C, Morlat P, et al. Determinants of delayed diagnosis of HIV infection in France, 1993-1995. AIDS 1998; 12 (7): 795-800. Epub 1998/06/10.

7.- MINSAL. Manual de procedimientos para la detección y diagnóstico de la infección por VIH. Santiago, Chile 2010. p. 223.

8.- MINSAL. Informe nacional sobre los progresos realizados en la aplicación del UNGASS Chile.
Informe Final. Santiago: Gobierno de Chile; 2010. p. 135.

9.- Schwarcz S, Richards T A, Frank H, Wenzel C, Chin Hsu L, Chin C S, et al. Identifying barriers to HIV testing: personal and contextual factors associated with late HIV testing. AIDS Care 2011: 1.

10.- Chadborn T R, Delpech V C, Sabin C A, Sinka K, Evans B G. The late diagnosis and consequent short-term mortality of HIV-infected heterosexuals (England and Wales, 2000-2004). AIDS 2006; 20 (18): 2371-9. Epub 2006/11/23.

11.- Krentz H B, Auld M C, Gill M J. The high cost of medical care for patients who present late (CD4 $<200$ cells/microL) with HIV infection. HIV Med 2004; 5 (2): 93-8. Epub 2004/03/12.

12.- Zolopa A, Andersen J, Powderly W, Sánchez A, Sanne I, Suckow C, et al. Early antiretroviral therapy reduces AIDS progression/death in individuals with acute opportunistic infections: a multicenter randomized strategy trial. PLoS One 2009; 4 (5): 18.

13.- Wood E, Kerr T, Marshall B D, Li K, Zhang R, Hogg R S, et al. Longitudinal community plasma HIV-1 RNA concentrations and incidence of HIV-1 among injecting drug users: prospective cohort study. Br Med J 2009; 338: b1649. Epub 2009/05/02.

14.- Donnell D, Baeten J M, Kiarie J, Thomas K K, Stevens W, Cohen C R, et al. Heterosexual HIV-1 transmission after initiation of antiretroviral therapy: a prospective cohort analysis. Lancet 2010; 375 (9731): 2092-8. Epub 2010/06/12.

15.- Fleishman J A, Yehia B R, Moore R D,
Gebo K A. The economic burden of late entry into medical care for patients with HIV infection. Med Care 2010; 48 (12): 1071-9.

16.- Glasman L R, Weinhardt L S, Difranceisco W, Hackl K L. Intentions to seek and accept an HIV test among men of Mexican descent in the Midwestern USA. AIDS Care 2010; 22 (6): 718-28. Epub 2010/05/15.

17.- Adler A, Mounier-Jack S, Coker R J. Late diagnosis of HIV in Europe: definitional and public health challenges. AIDS Care 2009; 21 (3): 284.

18.- Marks G, Crepaz N, Janssen R S. Estimating sexual transmission of HIV from persons aware and unaware that they are infected with the virus in the USA. AIDS 2006; 20 (10): 1447-50. Epub 2006/06/23.

19.- Mill J E, Jackson R C, Worthington C A, Archibald C P, Wong T, Myers T, et al. HIV testing and care in Canadian aboriginal youth: $\mathrm{A}$ community based mixed methods study. BMC Infect Dis 2008; 8.

20.- Trieu S L. Partner communication and factors associated with the decision to obtain an HIV test among Chinese/Chinese American community college students in Northern California. Dissertation Abstracts International: Section B: The Sciences and Engineering 2008; 69 (4-B): 2279.

21.- Ransom J E, Siler B, Peters R M, Maurer M J. Worry: Women's experience of HIV testing. Qualitative Health Research 2005; 15 (3): 382-93.

22.- Aho J, Nguyen V K, Diakite S, Sow A, Koushik A, Rashed S. High acceptability of 
HIV voluntary counselling and testing among female sex workers: impact of individual and social factors. HIV Med 2012; 13 (3): 156-65. Epub 2011/11/24.

23.- Barnabas Njozing N, Edin K E, Hurtig A K. 'When I get better I will do the test': Facilitators and barriers to HIV testing in Northwest Region of Cameroon with implications for TB and HIV AIDS control programmes. SAHARA J: journal of Social Aspects of HIV/AIDS Research Alliance/SAHARA, Human Sciences Research Council 2010; 7 (4): 24-32.

24.- Maman S, Mbwambo J, Hogan N M, Kilonzo G P, Sweat M. Women's barriers to HIV-1 testing and disclosure: challenges for HIV-1 voluntary counselling and testing. AIDS Care 2001; 13 (5): 595-603. Epub 2001/09/26.

25.- Njau B, Watt M, Ostermann J, Manongi R, Sikkema K. Perceived acceptability of homebased couples voluntary HIV counseling and testing in northern Tanzania. AIDS Care 2012; 24 (4): 413-9.

26.- Mitra D, Jacobsen M, O'Connor A, Pottie K, Tugwell P. Assessment of the decision support needs of women from HIV endemic countries regarding voluntary HIV testing in Canada. Patient Educ Couns 2006; 63 (3): 292-300.

27.- Nyanzi-Wakholi B, Lara AM, Watera C, Munderi P, Gilks C, Grosskurth H. The role of HIV testing, counselling, and treatment in coping with HIV/AIDS in Uganda: A qualitative analysis. AIDS Care 2009; 21 (7): 903-8.

28.- Myers T, Orr K W, Locker D, Jackson E A Factors affecting gay and bisexual men's decisions and intentions to seek HIV testing. Am J Public Health 1993; 83 (5): 701-4. Epub 1993/05/01.

29.- Spielberg F, Kurth A, Gorbach P M, Goldbaum G. Moving from apprehension to action: HIV counseling and testing preferences in three at-risk populations. AIDS Educ Prev 2001; 13 (6): 524-40. Epub 2002/01/17.

30.- Jurgensen M, Tuba M, Fylkesnes K, Blystad A The burden of knowing: balancing benefits and barriers in HIV testing decisions. a qualitative study from Zambia. BMC Health Serv Res 2012; 12: 2. Epub 2012/01/10.

31.- Daftary A, Padayatchi N, Padilla M. HIV testing and disclosure: A qualitative analysis of TB patients in South Africa. AIDS Care 2007; 19 (4): $572-7$.

32.- Sambisa W, Curtis S, Mishra V. AIDS stigma as an obstacle to uptake of HIV testing: Evidence from a Zimbabwean national population-based survey. AIDS Care 2010; 22 (2): 170-86.

33.- Castle S. Doubting the existence of AIDS: A barrier to voluntary HIV testing and counselling in urban Mali. Health Policy Planning 2003; 18 (2): 146-55.

34.- Bravo P, Edwards A, Rollnick S, Elwyn G.
Tough decisions faced by people living with HIV: a literature review of psychosocial problems. AIDS Reviews 2010; 12 (2): 76-88.

35.- de Wit J B, Adam P C. To test or not to test: psychosocial barriers to HIV testing in highincome countries. HIV Med 2008; 9 (Suppl 2): 20-2. Epub 2008/07/02.

36.- Fakoya I, Reynolds R, Caswell G, Shiripinda I. Barriers to HIV testing for migrant black Africans in Western Europe. HIV Medicine 2008; 9 (Suppl 2): 23-5.

37.- Carrizosa C M, Blumberg E J, Hovell M F, Martínez-Donate A P, García-González G, Lozada R, et al. Determinants and prevalence of late HIV testing in Tijuana, México. AIDS Patient Care STDS 2010; 24 (5): 333-40.

38.- Marks G, Crepaz N, Senterfitt J W, Janssen R S. Meta-analysis of high-risk sexual behavior in persons aware and unaware they are infected with HIV in the United States: implications for HIV prevention programs. J Acquir Immune Defic Syndr 2005; 39 (4): 446-53. Epub 2005/07/13.

39.- Araya A, Carrasco P, Loayza C, Fernández A-M, Pérez C, Lasso M. Necesidades educativas de un grupo de personas viviendo con VIH: diagnóstico basado en la perspectiva de los usuarios y de los profesionales que los atienden. Rev Med Chile 2013; 141 (5): 582-8. 